\title{
Taking the Pulse of US College Campuses with Location-Based Anonymous Mobile Apps
}

\author{
YANQIU WU, New York University Shanghai, Shanghai, China \\ TEHILA MINKUS, New York University, New York, USA \\ KEITH W. ROSS, New York University Shanghai, Shanghai, China; New York University, New York, USA
}

We deploy GPS hacking in conjunction with location-based mobile apps to passively survey users in targeted geographical regions. Specifically, we investigate surveying students at different college campuses with Yik Yak, an anonymous mobile app that is popular on US college campuses. In addition to being campus centric, Yik Yak's anonymity allows students to express themselves candidly without self-censorship.

We collect nearly 1.6 million Yik Yak messages ("yaks”) from a diverse set of 45 college campuses in the United States. We use natural language processing to determine the sentiment (positive, negative, or neutral) of all of the yaks. We employ supervised machine learning to predict the gender of the authors of the yaks and then analyze how sentiment differs among the two genders on college campuses. We also use supervised machine learning to classify all the yaks into nine topics and then investigate which topics are most popular throughout the US and how topic popularity varies on the different campuses. The results in this article provide significant insight into how campus culture and student's thinking varies among US colleges and universities.

CCS Concepts: • Computing methodologies $\rightarrow$ Machine learning; Supervised learning; Natural language processing; • Applied computing $\rightarrow$ Sociology;

Additional Key Words and Phrases: Social networks, data mining

ACM Reference format:

Yanqiu Wu, Tehila Minkus, and Keith W. Ross. 2017. Taking the Pulse of US College Campuses with LocationBased Anonymous Mobile Apps. ACM Trans. Intell. Syst. Technol. 9, 1, Article 12 (September 2017 ), 18 pages. http://dx.doi.org/10.1145/3078843

\section{INTRODUCTION}

I find that the three major administrative problems on a campus are sex for the students, athletics for the alumni, and parking for the faculty.

-Clark Kerr, first chancellor of UC Berkeley

What topics do college students discuss on campuses across the United States? What is the general sentiment-positive, negative, or neutral-on a college campus on any given day or throughout an academic year? Though researchers have traditionally explored students'

This work was supported in part by the NSF grant CNS-1318659.

Authors' addresses: Y. Wu, 200 Greene St, Jersey City, NJ 07311; email: yanqiu.wu@nyu.edu; T. Minkus, 10.054, 10th floor, 2 Metrotech, Brooklyn, NY 11201; email: tehila@nyu.edu; K. W. Ross, 1555 Century Avenue, Shanghai, China; email: keithwross@nyu.edu.

Permission to make digital or hard copies of all or part of this work for personal or classroom use is granted without fee provided that copies are not made or distributed for profit or commercial advantage and that copies bear this notice and the full citation on the first page. Copyrights for components of this work owned by others than the author(s) must be honored. Abstracting with credit is permitted. To copy otherwise, or republish, to post on servers or to redistribute to lists, requires prior specific permission and/or a fee. Request permissions from permissions@acm.org.

2017 Copyright is held by the owner/author(s). Publication rights licensed to ACM.

ACM 2157-6904/2017/09-ART12 \$15.00

http://dx.doi.org/10.1145/3078843

ACM Transactions on Intelligent Systems and Technology, Vol. 9, No. 1, Article 12. Publication date: September 2017. 
views and sentiment through surveys and focus groups (Kiesa et al. 2007; Ng et al. 2010), these approaches are expensive and hard to scale. Additionally, in a non-anonymous focus-group format, students may be reluctant to disclose their views about polarizing or taboo issues.

In this article, we explore how location-based anonymous apps can be leveraged to take the pulse of college campuses across the United States. Today, many of the most popular mobile appssuch as Waze, Yelp, and Tinder-are location based, that is, they provide information relevant to the user's current geographical position. The user's current geographical position is determined by the smartphone using GPS (with its constellation of satellites), AGPS, cellular networks, and Wifi. A user's current geographical position, simply referred to as the GPS position, is then made available to mobile app software through the smartphone's API.

It is well known, however, that a smartphone's GPS location can easily be faked using GPS hacking. With GPS hacking, a user physically in Paris can set its smartphone GPS coordinates to a specific street corner in Brooklyn. In this matter, the user in Paris can use Yelp to browse reviews of restaurants in Brooklyn, or use Waze to see current traffic conditions in Brooklyn. In this article, in lieu of expensive and labor-intensive surveys and focus groups, we explore how GPS hacking can be used in conjunction with the mobile app Yik Yak to take the pulse of college campuses.

Yik Yak was founded in late 2013 and has since gained significant traction on college campuses across the US (Mahler 2015). Yik Yak provides a simple message board to which users post short messages, called yaks, which are typically less than 200 characters. Yik Yak differs from traditional social networks in two respects. First, using the smartphone's GPS location, posts on Yik Yak are shared only to nearby users. In particular, a Yik Yak user only sees messages in a rectangular region centered at the user's current location, with the rectangular region typically covering a few square kilometers (Xue et al. 2016). The region is large enough to cover most US college campuses. The second way Yik Yak differs from traditional social networks is that it is anonymous. Up until recently, all Yik Yak posts were fully anonymous. More recently, Yik Yak modified its service so posts now carry pseudonymous usernames, which users may change as frequently as they like.

In this article, we use Yik Yak and GPS hacking to collect student posts from campuses across the US. We first develop an environment that carries out this data collection in a fully automated fashion. We then employ this environment to collect nearly 1.6 million yaks from 45 US colleges and universities. We also classify each of the 45 colleges into the following categories: Christian universities, liberal arts colleges, top-ranked universities, big-ten campuses, public universities, women's colleges, men's colleges, historically black colleges, and two-year college campuses. We then build a pipeline to categorize, compare, and analyze the data at each campus and in each category. We seek to answer the following questions:

-What is the general sentiment (positive or negative) on each of the 45 campuses? Does campus sentiment differ significantly among campuses or among campus categories? We use natural language processing (NLP) to address these questions.

-Is it possible to predict the gender of the author of an anonymous yak with good precision? If so, then how does the general sentiment compare for males and females on the 45 campuses? Is it possible to use gender prediction to partially de-anonymize Yik Yak posts?

- What are the most popular topics being discussed over Yik Yak? Do any topics dominate the discussions? What words are used within the topics? Are certain topics discussed more frequently for some campus categories? Are certain topics discussed more frequently in some US geographical regions?

- Are there correlations between certain topics? Are there correlations between certain topics and campus enrollments or campus admission rates? We examine these issues for the topics of dating and sex, academics, substance abuse, and politics and religion. 
Yik Yak's anonymity allows students to express themselves candidly without self-censorship. The methodology in this article can provide significant insight into how student's thinking and campus culture varies among US campuses.

This article is organized as follows. In Section 2, we provide a brief overview of Yik Yak. In Section 3, we describe our data collection methodology. In Section 4, we provide a sentiment analysis of the collected data. In Section 5, we carry out a gender analysis of the collected data. In Section 6, we explore topic analysis. In Section 7 we summarize related work and in Section 8, we conclude.

\section{ABOUT YIK YAK}

In addition to sharing yaks, Yik Yak users can view, upvote, downvote, and reply to others' yaks. By default, a Yik Yak user views the yaks within a region of its geographical location.

Yik Yak employs community-driven moderation based on downvotes. Specifically, a yak that receives five negative ratings from other users is removed from the stream of yaks. Additionally, Yik Yak blocks posts within a certain radius of high schools and grade schools, an attempt to curtail digital bullying on Yik Yak (Graber 2014).

Yik Yak is particularly popular among students on college and university campuses. Much media coverage has focused on Yik Yak's potential as a platform for bullying and hate-speech (Mahler 2015), and a handful of universities have blocked access to the app on their networks (Rubelke 2015).

However, some brands view Yik Yak as an avenue to engage with the coveted millennial demographic, which comprises an estimated 98\% of Yik Yak's user base (Lella 2015). In particular, the BBC recently engaged with Yik Yak users during the 2015 Canadian presidential elections. BBC also solicited contributions from Yik Yak users during a special week of mental health coverage. Journalists found Yik Yak effective for eliciting honest responses on sensitive topics (Bilton 2016).

\section{DATA COLLECTION}

\subsection{Yak Retrieval}

One approach to collecting yaks is to take screen shots and use optical character recognition to extract the yaks. Although this process can be largely automated with existing task automation tools for smartphones, it is slow and prone to errors. We instead used the Yik Yak API to retrieve the yaks at the 45 campuses.

We set up a Python bot and employed GPS hacking to retrieve yaks. For each of the 45 campuses, we sent a request for recent yaks once per hour, yielding the 100 most recent yaks from that location. Because most yaks are visible for more than an hour, and thus were retrieved multiple times, we had to remove all duplicates from the dataset.

The Yik Yak API we used was based on the YakGrabber library (Gupta 2015), which we modified to accommodate for batch inputs of several target locations. Our library emulated an older version of the Yik Yak application for Android, building custom URLs to retrieve data for specific parameters. (The updated version of the Yik Yak application employs additional measures, making it more difficult to intercept and replay its communications, such as pinned SSL certificates and cryptographically hashed timestamps. While older versions of the application may no longer post yaks, Yik Yak's servers still allow read-only access for older versions.) We sent HTTP GET requests, and the Yik Yak endpoint returned the yaks in JSON (JavaScript Object Notation) format. We took care to rate-limit our requests by sending only one request per minute, thus avoiding overloading Yik Yak's servers. 
Table 1. Categories, Campuses, and Number of Yaks Collected at Each Campus

\begin{tabular}{|c|c|c|}
\hline Christian universities & Big Ten campuses & \\
\hline Brigham Young U 39,919, & Indiana University, 44,647 & Men's colleges \\
\hline Liberty University, 8,072 & Michigan State University, 103,037 & HampdenSydney College, 4,691 \\
\hline \multirow[t]{2}{*}{ Grand Canyon University, 41,637 } & Northwestern University, 38,313 & Saint John's University, 1,533 \\
\hline & Ohio State U, Main Campus, 102,167 & Wabash College, 1,464 \\
\hline Liberal arts college campuses & Penn State, Main Campus, 65,454 & \\
\hline Bowdoin College, 7,150 & Purdue University, 65,815 & \\
\hline Middlebury College, 5,474 & U of Illinois, Urbana-Champaign, 61,470 & Historically black colleges \\
\hline Swarthmore College, 9,311 & University of Iowa, 42,053 & Florida A\&M University, 68,881 \\
\hline \multirow[t]{2}{*}{ Williams College, 8,071} & U of Michigan, Ann Arbor, 74,330 & Howard University, 19,500 \\
\hline & U of Minnesota, Twin Cities, 55,090 & Jackson State University, 3,642 \\
\hline Top-ranked universities & University of Nebraska, Lincoln, 23,237 & North Carolina A\&T State U, 8,530 \\
\hline Columbia University, 15,096 & & Texas Southern University, 38,760 \\
\hline \multicolumn{3}{|l|}{ Harvard University, 21,526 } \\
\hline Princeton University, 26,058 & Public universities & \\
\hline Stanford University, 19,042 & Arizona State U, Tempe, 45,209 & Two year college campuses \\
\hline \multirow[t]{2}{*}{ Yale University, 12,667} & Miami Dade College, 6,056 & Austin Community College, 63,778 \\
\hline & Rutgers U, New Brunswick, 34,578 & East Los Angeles College, 3,698 \\
\hline Women's colleges & Texas A\&M, College Station, 121,481 & Ivy Tech Community College, 3,282 \\
\hline Bryn Mawr College, 14,916 & University of Central Florida, 118,636 & Portland Community College, 1,211 \\
\hline Scripps College, 11,003 & University of Florida, 34,848 & \\
\hline Wellesley College, 18,938 & University of Texas at Austin, 65,462 & \\
\hline
\end{tabular}

\subsection{Campus Categories}

As our goal is to get compare students' thinking and campus culture across the United States, we selected 45 universities diverse in character and geographic location. Specifically, we consider nine categories of colleges and universities and universities: Christian universities, liberal arts colleges, top-ranked universities, big-ten campuses, public universities, women's colleges, men's colleges, historically black colleges, and two-year college campuses. We choose these categories, because they are diverse yet collectively include most of the universities in the US.

From each of these categories, we selected several colleges while aiming for geographic diversity. In selecting colleges from these categories, we prioritized campuses with high enrollment, based on the figures provided by the National Center for Education Statistics report (Education Statist ics 2014). For top-ranked universities, we drew universities from the U.S. News and World Report annual college ranking. Table 1 lists the categories and campuses.

\subsection{Dataset}

We hired workers on Amazon Mechanical Turk to quickly and cheaply determine the GPS coordinates of each university. We collected a corpus of 1,579,733 yaks from the 45 US university campuses from 21 January 2016 to 20 June 2016. Each college in 1 is followed by the total number of yaks collected at that college. Of these 1,579,733 yaks, a little over 100,000 had handles (pseudonyms). We also mined publicly available data from CollegeData about each campus.

\subsection{Legal and Ethical Considerations}

We applied for IRB (Institutional Review Board) approval and were found to be exempt. We also limited our crawlers to ensure that the Yik Yak servers would not be overloaded.

\section{SENTIMENT ANALYSIS}

In this section, we analyze and compare the sentiment of the yaks across all 45 universities. If one accepts that the candid and anonymous posts on Yik Yak reflect student's thinking and feelings, then the results here can help determine at which types of schools students are, in aggregate, 
happier. For both sentiment and gender analysis, we remove yaks that have only one or two words for both training and validation, since these yaks are generally meaningless and irrelevant.

\subsection{Lexicon-Based Classification}

There are two broad approaches that can be taken for sentiment analysis: supervised machinelearning approaches and un-supervised lexicon-based approaches. For supervised sentiment analysis, we first need to label a subset of the yaks as positive, negative, or neutral. Then using this labeled data, we use supervised machine-learning algorithms to classify the sentiment of the remaining yaks. In the un-supervised lexicon-based approach, we use emotional dictionaries that classify words and phrases in terms of emotional content. An emotional score is then given to each yak based on the words and phrases in the yak. As the supervised approach requires significant effort to create the labeled data set, and also suffers from the subjectivity of the labelers, we use the un-supervised lexicon-based approach in this article.

Many sentiment analysis algorithms are focused on supervised, machine-learning approaches. In this article, we use an unsupervised, lexicon-based approach to measure the emotional intensity contained in yaks across the 45 university campuses. Specifically, we use a lexicon-based approach based on the LIWC (Linguistic Inquiry and Word Count) program, which is publicly available with an open API (Paltoglou and Thelwall 2012). The LIWC program, built with the aid of linguists and psychologists, classifies tokens in terms of their emotional content. For a given text, the algorithm extracts the polarity and intensity of words belonging to the emotional dictionary. Words five words before and after the emotion terms are checked for negators (e.g., not), intensifiers (e.g., very), or diminishers (e.g., little). Negators will reverse the emotion weights of emotion terms but reduce the absolute values by 1 (e.g., -3 becomes 2 and 3 become -2 ). Intensifiers will increase/decrease the emotion weights of positive/negative terms by its intensity weight. Diminishers, intuitively, do the opposite of intensifiers. The classifier outputs each yak as positive, negative, or neutral.

\subsection{Sentiment Results}

Since the algorithm does not recognize emoticons, we transfer emoticons in the yaks into actual words based on a Python library called emoji (Kim and Wurster 2015). Then we perform the lexicon-based sentiment analysis over all the yaks in our Yik Yak dataset. Table 2 shows examples of five positive yaks and five negative yaks.

For each college, we define two sentiment metrics: sentiment ratio, which is the ratio of the number of negatively classified yaks to the number of positively classified yaks, and emotion level, which is the sum of the positively classified yaks and the negatively classified yaks divided by the total number of yaks for that college. Figure 1 shows a scatter plot of the 45 universities with the sentiment ratio on the $x$-axis and the emotion level on the $y$-axis. From Figure 1, we see that all 45 schools have more negative posts than positive posts. We further see that the sentiment ratio varies greatly from campus to another, ranging from 1.22 to 1.66 . The emotion level also varies significantly from school to school, ranging from $53 \%$ to $69 \%$. However, we see from Figure 1 that most colleges have an emotion level of $60 \%$ to $70 \%$, thus for most colleges, $30 \%$ to $40 \%$ of the yaks are neutral. Figure 1 also shows the MSE regression line. We see that schools with higher sentiment ratios (i.e., more negative) tend to have a higher emotion level, although the correlation is not statistically significant.

Figure 2(a) displays sentiment ratios by school category. For a given category, there is one horizontal bar, with the left-side of the bar being the lowest sentiment ratio among all the schools in the category and the right-side of the bar being the highest sentiment ratio among all the schools 
Table 2. Examples of Five Yaks Classified as Having Positive Sentiment, and Five Yaks Classified as Having Negative Sentiment

\begin{tabular}{cl}
\hline Sentiment & \multicolumn{1}{c}{ Sample Yak } \\
\hline positive & Handles are fucking amazing!!!! \\
positive & $\begin{array}{l}\text { Talking to a match on tinder a million time out of my league, and it's going } \\
\text { great. Pretty successful day! }\end{array}$ \\
positive & $\begin{array}{l}\text { Sitting on the patio. Glass of wine. Listening to a young girl talk about her } \\
\text { upcoming wedding. Congrats! }\end{array}$ \\
positive & Congrats to all of the graduates today!! \\
positive & Good luck on your comps seniors! \\
negative & The fact that you can't retake tests when you're out sick is incredibly shitty \\
negative & Insomnia, heartbreak, anxiety....what do I do? \\
negative & The rate on dollars are so shitty rn I'm crying! Fuck America exchange \\
negative & I'll be so sad if Hillary wins in PA. pensive face \\
negative & It's so fucking annoying spending every weekend stressed out about doing my \\
& homework
\end{tabular}

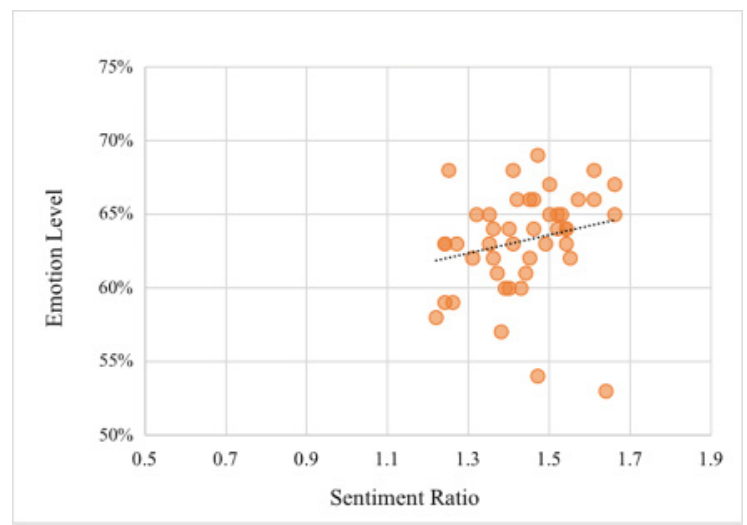

Fig. 1. Scatter plot of 45 universities with respect to sentiment and emotion

in the category. Each bar also has a vertical line indicating the average sentiment ration in the category. Figure 2(b) provides a similar visualization for emotion levels.

Surprisingly (at least to the authors), Figure 2(a) clearly shows that the Christian universities are the most positive among the nine university categories. Moreover, all three Christian universities have almost the same sentiment ratio. The majority of the students at these universities are devout Christians. We can conjecture that these students have a relatively positive outlook on life, although we offer no means to validate this conjecture. One might conjecture that large universities would generally be relatively negative; however, the two Christian schools Brigham Young University and Grand Canyon University both have large student bodies, with about 30,000 and 20,000 on-campus students, respectively. Thus there does not seem to be a correlation between the size of a school the and the sentiment of the students.

After the Christian schools, the liberal arts colleges are the most positive. In fact, all four of the liberal arts colleges are more positive than all of the schools in the big-ten, top-ranked, historically black, women's college, and men's college categories. The liberal arts colleges considered here are all small private schools with high tuition and high faculty-to-student and staff-to-student ratios. 


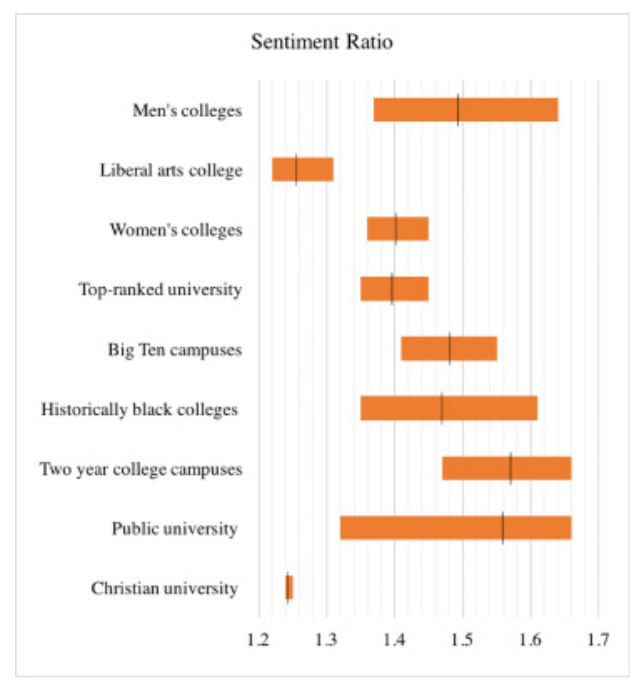

(a) Sentiment Ratio

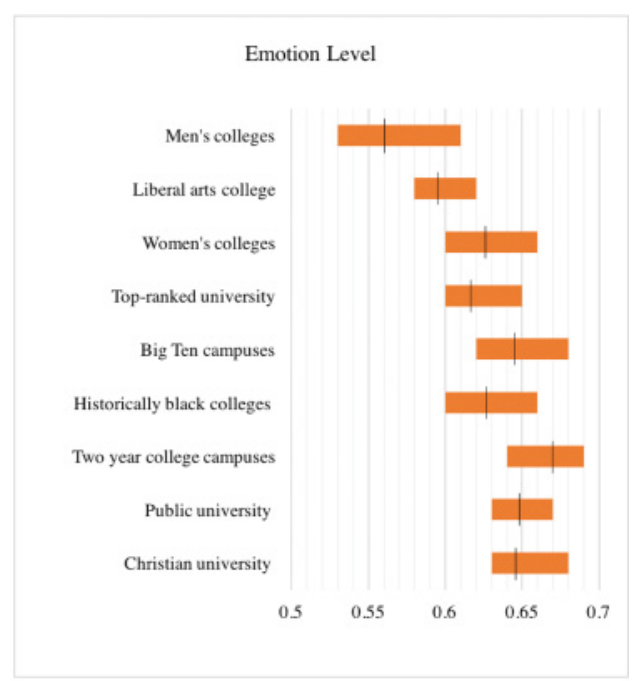

(b) Emotion Level

Fig. 2. Sentiment ratios and emotion levels by school category.

On the other hand, the big-ten schools have relatively low in-state tuition and generally have larger classes and lower staff-to-student ratios. Again, without any ground truth to validate, we can conjecture that higher faculty and staff to student ratios lead to a happier student body in aggregate. Following the Christian and the liberal-arts schools, the top-ranked universities and women's colleges are the most positive.

On average the two-year colleges are the most negative. Again without any attempt to validate the claim, this may be because students at two-year universities often come from less privileged economic backgrounds as compared to their four-year college counterparts. Alternatively, this may also be because the two-year colleges are commuter schools, whereas the four-year colleges considered in this study are primarily residential schools.

The emotion-level results, shown in Figure 2(b), are not quite as striking. There is generally a lot of variety within each category. The men's colleges on average are the least emotional, and two-year colleges on average are the most emotional.

\section{GENDER PREDICTION AND ANALYSIS}

It is of interest to see if we can accurately predict the gender of the author of a yak for two reasons. First, if we can accurately classify the authors as male or female, then we can perform a genderbased analysis of sentiment and topics. Second, accurate gender classification would be one step further towards de-anonymizing the yaks. Indeed, in an earlier article, it was shown that it is possible to determine the location from which a yak was posted to the granularity at a dorm building (Xue et al. 2016). By combining known location with known gender, one can further narrow down the potential authors of a yak. De-anonymization puts into question the main feature of Yik Yak and other related anonymous mobile apps.

\subsection{Labeling the Gender of Yaks}

To classify the yaks as male or female using supervised learning, a subset of the yaks need to be labeled. Recall that a fraction of the yaks have handles (pseudonyms). Many of these handles 
Table 3. Stylometric Features

\begin{tabular}{ll}
\hline Category & \\
\hline Length & number of words in yak \\
Emoji & number of emojis in yak \\
Digits & number of digits in yak \\
Special character & number of special characters \\
Punctuation & number of ? and ! in yak \\
Word shape & number of words with all upper case/all lower case/first letter upper \\
& case/CamalCase \\
Word length & number of words that have more than 20 characters \\
\hline
\end{tabular}

reveal gender information, for example, handles like "Princessshh," "missmango," and "crystalbeth" have a very high probability of being created by female users. Similarly, "SammyDavisJr," "BlueEyesWhiteGuy," and "kingfkush" are very likely created by male users. We hired workers from Amazon Mechanical Turk (AMT) to quickly and cheaply classify handles as female, male, or not sure. Each yak was classified by five different workers on AMT. We declare its final gender label as not sure unless three or more workers vote the same gender and no contradictions occur with the remaining workers. For example, a handle would be labeled as male if and only if the five votes are MMMNN, MMMMN, or MMMMM, with $\mathrm{M}$ representing male and $\mathrm{N}$ representing not sure. MMMMF would be labeled as not sure.

\subsection{Preprocessing for Prediction}

We use two kinds of features, bag-of-words features and stylometric features, for gender prediction. Details about the stylometric features can be found in Table 3. To ensure the quality and reliability of our feature choices, the following data pre-processing steps are applied:

-We group phrases and words with their corresponding abbreviations. For example, we group "bf" with "boyfriend," "hw" with "homework," "to be honest" with "tbh," and "oh my god" with "omg."

-We group contractions such as "will not" with "won't” and "does not" with "doesn't." We treat each grouping as one word.

-We classify punctuation into three classes: "?," "!," and "p." The "p" class covers everything else, including comma, period, and punctuation strings.

-We stem every word.

After performing the above steps, we have 3,754 yaks labeled as female and 13,458 yaks labeled as male. To deal with this imbalanced data, we re-sample the minority class by duplicating entries. To avoid over-fitting, we over-sample after cross-validation, that is, after we leave the validation set out of the training loop (Japkowicz 2000). In this way, we can make sure that no duplicated entries will be both trained and validated. After testing a number of algorithms, we use Support Vector Machines (SVMs) and report averaged 10-fold cross-validation test results.

\subsection{Gender Prediction Results}

Table 4 summarizes gender classification results when using only bag-of-words features. We control the value of recall by not predicting data points that are within a certain distance to the optimal hyper plane. We see that we have nearly $59 \%$ precision. If we consider only the $15 \%$ of yaks that are furthest from the SVM hyperplane, then we can have nearly $71 \%$ precision. Thus, using 
Table 4. Gender Classification

Results with Bag-of-Words

Features

\begin{tabular}{cc}
\hline \multicolumn{2}{c}{ Gender Classification } \\
Approximate Recall & Precision \\
\hline $100 \%$ & $59.1 \%$ \\
$50 \%$ & $61.1 \%$ \\
$25 \%$ & $64.9 \%$ \\
$15 \%$ & $68.2 \%$ \\
$5 \%$ & $74.4 \%$ \\
\hline
\end{tabular}

Table 5. Ten Most Weighted Feature Words

\begin{tabular}{|c|c|}
\hline \multicolumn{2}{|c|}{ Feature Words } \\
\hline Male Class & Female Class \\
\hline girlfriend & boyfriend \\
\hline man & tomorrow \\
\hline $\begin{array}{l}\text { spring } \\
\text { fuck }\end{array}$ & \\
\hline $\begin{array}{l}\text { Iuck } \\
\text { gal/girl }\end{array}$ & $\begin{array}{l}\text { someone } \\
\text { cat }\end{array}$ \\
\hline show & bed \\
\hline yik & drink \\
\hline shit & thing \\
\hline know & (경 \\
\hline point & stop \\
\hline
\end{tabular}

Table 6. Gender Classification Results with Different Features (100\% Recall)

\begin{tabular}{cc}
\hline Features & Precision \\
\hline Bag-of-words features & $59.1 \%$ \\
Stylometric & $51.7 \%$ \\
Bag-of-words + stylometric & $57.0 \%$ \\
\hline
\end{tabular}

word-usage alone, we can make reasonably accurate predictions about whether the author of a yak is male or female.

Each gender class has its own set of most highly weighted feature words. Table 5 lists the top 10 feature words for each class. It is interesting that the most distinguishing predictor of whether a yak is authored by a male is the presence of the word "girlfriend," whereas symmetrically the most distinguishing predictor for a female is the presence of the word "boyfriend." We also note that male yaks are also distinguished by the use of profanity and female yaks by the use of emoticons.

We also apply SVM using stylometric features. Furthermore, we combine the normalized realvalued stylometric features with binary bag-of-words features. We normalize the stylometric feature values by dividing each value by the 2 -norm of its stylometric feature array. In this way, every stylometric feature value is bounded by 0 and 1 .

Table 6 summarizes the classification results. In this case, we only compare precision for $100 \%$ recall. We can easily see that stylometric features work poorly. Women and men in aggregate appear to use similar styles for short messages like yaks. 


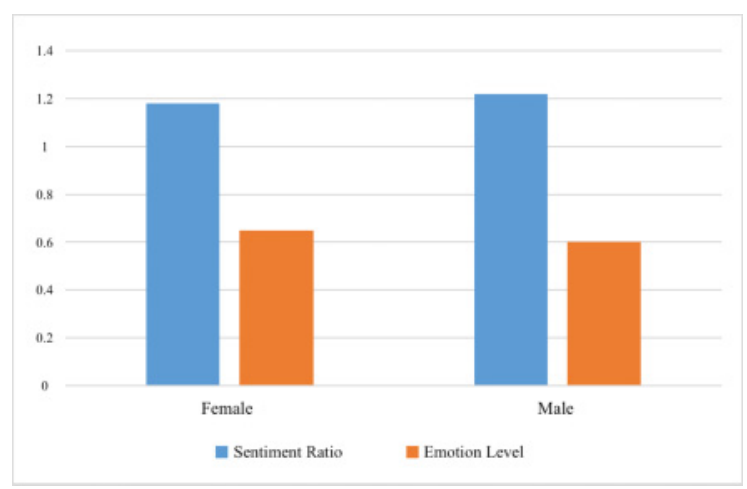

Fig. 3. Sentiment ratio and emotion level for females and males.

\subsection{Gender Sentiment}

Using only the yaks for which the author gender was manually labeled by AMT workers, we used the lexicon-based sentiment analysis tool to analyze the sentiment of the two genders. Female students and male students seem to have very similar sentiment ratios and emotion levels, as shown in Figure 3. However, we must emphasize that this observation is being drawn from subset of yaks, namely those that have revealed their genders through their handles. Interestingly, students who reveal their gender are on average more positive than students who do not reveal their gender.

\section{TOPIC ANALYSIS}

For the topic analysis, we decided to focus on a one-month period (January 21 to February 21, 2016) during which the presidential primaries were receiving intense media coverage. This gives a sub-corpus from the dataset of nearly 500,000 yaks from the 45 schools. We manually examined 1,000 yaks to develop a codebook of eight topics. Table 7 lists the eight topics.

We randomly sampled 10,064 yaks from our dataset (roughly $2 \%$ overall). We then posted a task on Amazon Mechanical Turk, asking the workers to classify each yak into one of the eight topics shown in Table 7. We included a ninth topic for "I don't know," since some yaks are hard to understand. If at least two of three workers chose the same topic for a single yak, then we assigned the yak to that topic. There was consensus for $85 \%$ of the yaks. The frequency for each of the topics is shown in Table 7.

We can draw some preliminary conclusions based on this sample of 10,064 yaks. Notably, dating and sex comprise a large portion of conversations on Yik Yak. Popular culture and academics are widely discussed, as are local inquiries and announcements. There is a fair bit of discussion about health and substance issues, and political yaks have a consistent presence as well. However, counter to claims by many media outlets and university administrators, discriminatory and racist yaks are not very prevalent in our sample. This would seem to bolster claims by Yik Yak that their moderation and downvoting policies are effective.

Figure 4 shows the frequent terms from some of the topics. These word clouds help to understand the content in the yaks in these topics.

\subsection{Scaling Up the Analysis}

To learn more broadly about students' yaks across the United States, we employ machine-learned classifiers to label all the yaks in the one-month dataset. We used the ground-truth topics established above to train machine-learning classifiers for the most interesting topics: dating and sex, 
Table 7. Topics and Their Occurrence within the Sample Set of 10,064 Yaks. For $15 \%$ of the Dataset, There Was No Consensus on a Single Category

\begin{tabular}{|c|c|c|}
\hline Topic & $\%$ of sample & Sample Yak \\
\hline$\overline{\text { Dating and sex }}$ & 23 & $\begin{array}{l}\text { Any girls looking for a late night make out } \\
\text { session? }\end{array}$ \\
\hline $\begin{array}{l}\text { Local life, weather, } \\
\text { announcements }\end{array}$ & 15 & Are there any bus routes to Reed Arena? \\
\hline Culture, tech, and sports & 12 & $\begin{array}{l}\text { Thomas Bryant is the only thing ESPN can talk } \\
\text { about apparently }\end{array}$ \\
\hline Academics and careers & 11 & $\begin{array}{l}\text { Does anyone wanna work on the Stats project for } \\
\text { Samantha Russels class together? Need some help }\end{array}$ \\
\hline Health, drugs, alcohol & 7 & $\begin{array}{l}\text { With the } 2 \text { beers I burned off, I can have another } 2 \\
\text { beers! }\end{array}$ \\
\hline Politics and religion & 5 & $\begin{array}{l}\text { It's just like the high schoolers on the news said. } \\
\text { Donald Trump: make America hate again }\end{array}$ \\
\hline Friends & 4 & $\begin{array}{l}\text { I miss my college friends so much. Don't take } \\
\text { your friends for granted. }\end{array}$ \\
\hline Diversity/discrimination & 1 & $\begin{array}{l}\text { Some of these international students act like } \\
\text { they've never lived in a civilized society before }\end{array}$ \\
\hline I don't know & 6 & *trombone* *oven door slamming ${ }^{*}$ \\
\hline
\end{tabular}

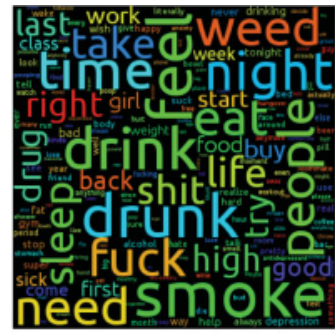

(a) Health, drugs, and alcohol (b) Diversity and discrimination

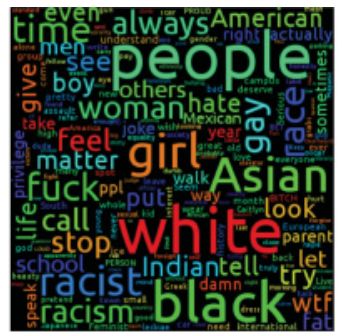

Fig. 4. Word clouds depict the frequent words in each to topic.

academics, politics and religion, and alcohol and substances. (We were unable to train a classifier for racist/bullying yaks, since they were so rare in our ground-truth training set.)

After testing a number of algorithms, we used Random Forest and Decision Tree algorithms for the different categories. To determine each classifier's performance, we ran the selected algorithm with 10 -fold cross-validation. Average precision ranged from $86 \%$ to $92 \%$, and average recall ranged from $87 \%$ to $97 \%$. For features, we removed stopwords and generated word-count vectors and TFIDF (term frequency-inverse document frequency) vectors. We did not use $n$-grams, since they degraded precision and recall on our test sets.

\subsection{Analyze Yaks at Scale}

After developing a pipeline to categorize yaks, we analyze the dataset to answer a few important questions. How do campuses differ in yakking behaviors? Do certain campus characteristics (e.g., enrollment, selective admissions rates, demographics, geographic region) correlate to topic popularity? 
In this section, we analyze yaks labeled by our machine-learned classifiers. We note that all ANOVA (analysis of variance) analyses mentioned in this section are significant at the $p \leq 0.05$ level and are followed by post-hoc Tukey HSD (honest significant difference) tests. All correlations mentioned in this section are significant at the $p \leq 0.05$ level after a Holm-Sidak correction for multiple comparisons, unless otherwise specified. In Figure 6, the $r$-value is the Pearson's correlation coefficient, the blue line is the regression line, and the $p$-value is the two-tailed $p$-value. The Pearson correlation coefficient measures the linear relationship between two datasets.

\subsection{Dating and Sex}

The most popular topic in our dataset is dating and sex. How does its incidence differ across colleges, regions, and campus types?

We see from Figure 5 that men's colleges yak the least frequently about dating and sex. Women's colleges yak more frequently but still relatively less than most of the campus categories. One possible interpretation is that single-gender campuses yak about dating and sex at lower rates, because dating prospects are greatly limited for heterosexual students at such campuses. Historically black colleges, two-year colleges, public universities, and Christian universities tend to yak the most about dating and sex. The campus with the highest rate of yakking about dating and sex, Jackson State University, had $39.1 \%$ of its total yaks labeled with this topic. In contrast, the campus that yakked least about dating and sex (Hampden-Sydney College) had only $6.6 \%$ of its total yaks labeled with this topic.

Regional differences were also evident, as backed up by an ANOVA analysis. Campuses located in the west had a statistically significant higher rate of mentioning dating and sex (see Figure 5), with $27.1 \%$ of their yaks labeled with this topic. Meanwhile, only $18.6 \%$ of yaks from northeastern campuses were related to dating and sex. The south and midwest fell in the middle, with respective dating-and-sex yak rates of $24.7 \%$ and $21.5 \%$.

Another trend we observe is, in general, students yak more about dating and sex at campuses with higher enrollment. Figure 6(a) demonstrates this relationship (though it is not statistically significant). It is possible that students who plan to engage in a vibrant dating scene choose to attend larger campuses, which would explain the difference.

\subsection{Academics}

All campus types (e.g., public, Christian, two-year, etc.) have academic-yak rates between $5 \%$ and $10 \%$. Interestingly, the two school categories with the most positive sentiment ratio-Christian schools and liberal arts colleges-are also the two categories with the lowest yaking rates about academics. One can conjecture that the schools at which students are the least stressed about academics (for whatever reason) are also the schools where the students are the happiest. All regions (northeast, south, midwest, and west) yakked about academics at rates between $7 \%$ and $9 \%$, with no statistically significant differences between regions. Interestingly, we also find that campuses with higher rates of yakking about dating and sex had lower rates of academic-related yaks at a statistically significant level (see Figure 6(c)). Presumably, students who are more involved in the dating scene maintain lower levels of academic investment.

We also examined relationships between academic yak rates and university characteristics, such as average SAT scores, acceptance rates, and demographic composition of student bodies. However, we found no evidence for statistically significant correlations.

\subsection{Substance Use}

The widespread use and abuse of alcohol and drugs on US college campuses is an important issue. Is this behavior reflected in yaks? Which campuses talk about alcohol and/or drugs most? To measure 


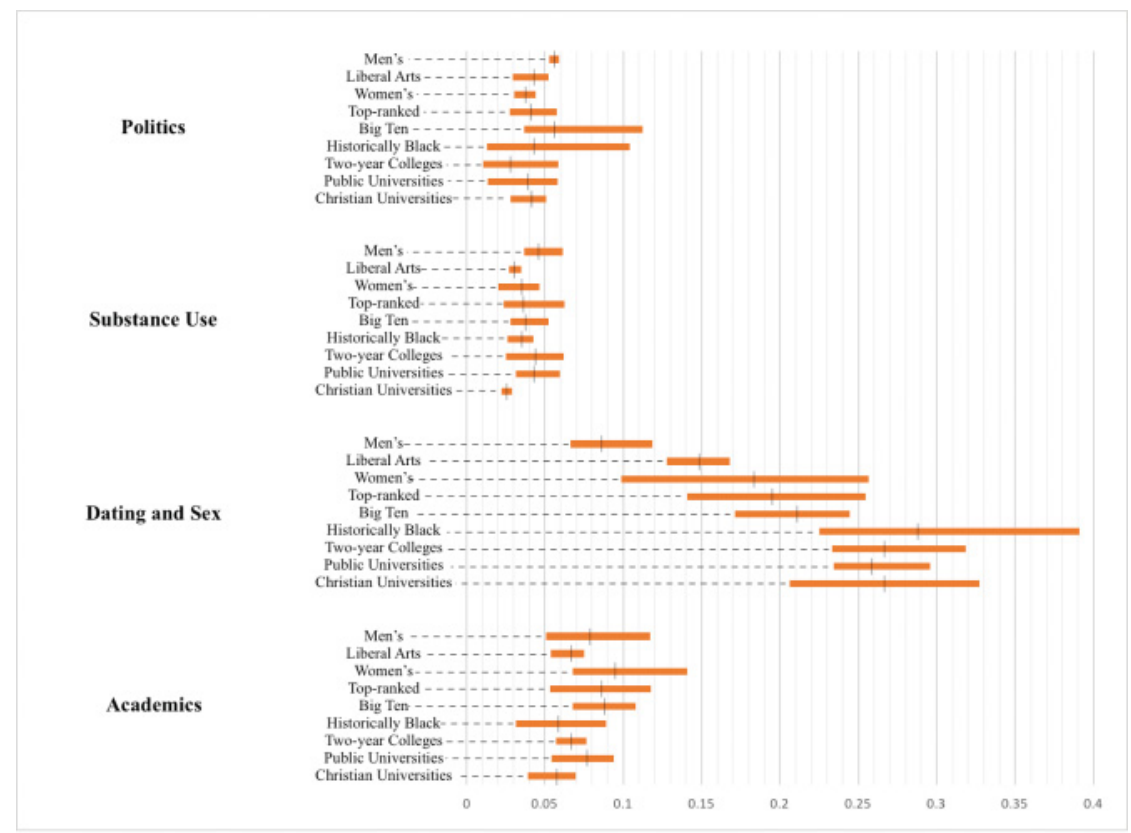

(a) By institution type

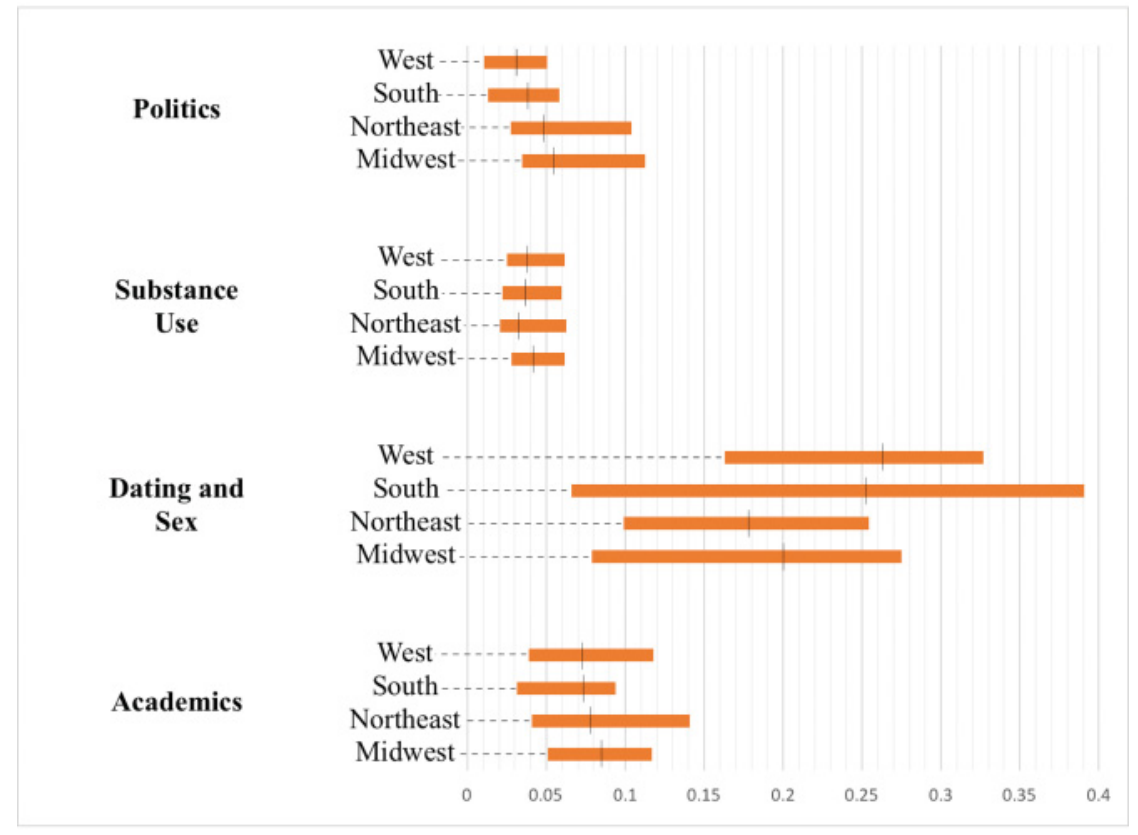

(b) By region

Fig. 5. Topic analysis: By campus category and by region. 


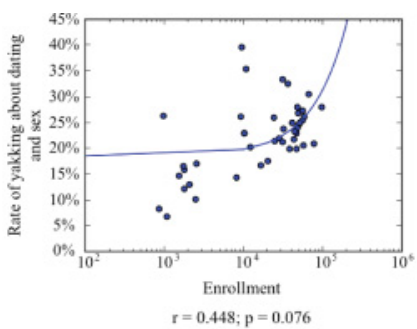

(a) Dating and sex vs. campus enrollment

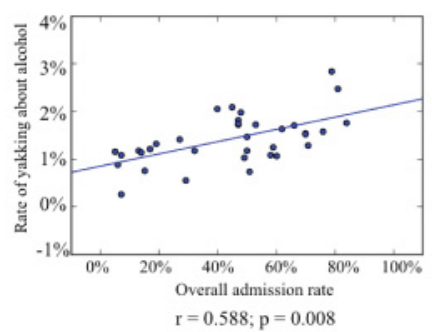

(b) Alcohol vs. campus admission rate

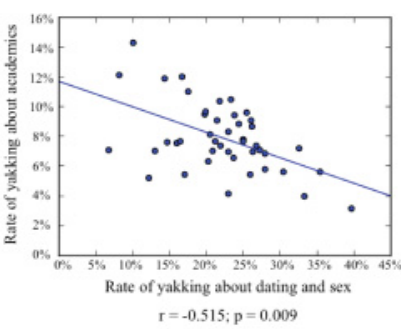

(c) Dating and sex yaks vs. academic yaks

Fig. 6. The popularity of topics when compared with campus characteristics.

this more carefully, we submitted each yak that had been automatically labeled as substance related to workers on Mechanical Turk to determine whether it discussed alcohol, drugs, or something else. After obtaining rates of yakking about alcohol and drugs (respectively) for each school, we calculated correlations with school types, regions, and other factors.

As Figure 6(b) shows, rates of alcohol-related discussions vary across campuses. In our analysis, one notable factor correlated with rates of substance-related yakking: campus admission rates. Specifically, campuses that accepted fewer applicants had statistically significant lower rates of alcohol-related yakking. A similar relationship was visible with respect to SAT scores: higher average SAT scores were associated with lower rates of substance-related yaks, although not at a statistically significant level. The lower rate of alcohol-related yakking at more selective institutions lends itself to multiple interpretations. Perhaps the students are more motivated to excel, and thus less likely to engage in drugs and alcohol, which may adversely impact their academic performance.

Regions also exhibited different rates of alcohol-related yakking in an ANOVA analysis: southern campuses yakked more about alcohol than those from any other region.

\subsection{Politics and Religion}

Recall that, for topic analysis, we are considering the yaks for a one-month period (January 21 to February 21,2016) during which the presidential primaries were receiving intense media coverage. As we shown in Table 7, politics and religion are fairly popular on Yik Yak, with approximately $5 \%$ of all yaks in our sample set falling into the politics/religion topic. Which campuses yak the most about politics during the time spanned by our dataset? The campus with the most political chatter was University of Iowa, which is located in the state with the first caucus or primary. In general, the campuses with higher rates of political chatter are located in states with earlier primaries. An ANOVA analysis of regions with respect to politics-and-religion discussion rates reveal that midwestern campuses were most likely to discuss politics and religion. It is likely that intense campaigning in early-primary midwestern states leads to higher rates of yakking about politics.

Which political candidates garnered the most discussion on Yik Yak? By searching for candidates using their names as keywords, we found that Bernie Sanders and Donald Trump each were mentioned in approximately $1 \%$ of the yaks; Hillary Clinton was mentioned in $0.5 \%$ of yaks; and the rest of the primary candidates were each mentioned in less than $0.2 \%$ of yaks.

Interestingly, the proportion of yaks about each candidate on campuses did not correlate with state primary results. This was because virtually all campuses' discussions about candidates were dominated by chatter about Bernie Sanders and Donald Trump. Even in states where another 
candidate won the majority of the popular vote and/or delegate count, Trump and Sanders were still discussed at higher rates than the actual winner.

\section{RELATED WORK}

\subsection{Sentiment Analysis}

Sentiment analysis has previously been applied to different types of social media such as blogs, Twitter, and Facebook. Pang et al. (2002) performed sentiment analysis on reviews based on machine-learning approaches. Agarwal et al. (2011) introduced POS (part-of-speech)-specific prior polarity features and tree representation features for sentiment analysis on tweets based on Dictionary of Affect in Language (Whissel 1989) and extended it using WordNet. However, their supervised algorithm requires labor-intensive manual labeling of the Twitter data.

Paltoglou and Thelwall (2012) implemented an unsupervised sentiment classifier based on the LIWC software (Pennebaker et al. 2001). In their experiments on the Digg and MySpace dataset, the lexicon-based classifier outperforms other supervised approaches (SVMs and Naive Bayes). However, their sentiment analysis did not target US college campuses. To our knowledge, this is the only study that uses social media to investigate the sentiment on different college campuses.

\subsection{Gender Prediction}

Predicting genders of blog authors and social media users from text has been studied by several researchers. The problem is very important in the field of author profiling and de-anonymization. For long texts, such as fiction books and blogs, $76 \%-80 \%$ accuracy has been achieved (Koppel et al. 2003; Argamon et al. 2009). Because the texts are much longer than a yak, they were able to obtain relatively high accuracy rates.

Gender prediction has also been performed for Twitter, which is similar to Yik Yak in post length. Rao et al. (2010) input $n$-gram features and sociolinguistic features, such as emoticon usage and character repetitions, into SVMs for gender classification. They reported an accuracy of $71.8 \%$ using sociolinguistic features, an accuracy of $68.7 \%$ when using $n$-gram features, and an accuracy of $72.3 \%$ when combining $n$-gram-features with sociolinguistic features. However, they did not make their predictions based on a single tweet but instead on the aggregation of tweets from the same author handle. Our gender prediction study differs in that we study the more challenging problem of predicting gender based on a single short yak, which typically ranges from 50 to 200 characters in length.

\subsection{Topic Analysis in Yik Yak}

Though Yik Yak is relatively new, it has gained some attention from the research community. McKenzie et al. (2015) compared Yik Yak to Twitter. They found that Yik Yak topics differ from and are more localized than Twitter topics. However, they only explored one square mile in Los Angeles, so their conclusions are not generalizable and do not explore differences among campuses.

Northcut (2015) manually examined screenshots of 319 yaks and categorized them into four intentions: shock, joke, inquire, and emote. Heston and Birnholtz (2016) collected yaks from 35 universities for multiple months and categorized the intent of the posts. By hand-coding 1,800 yaks, they found that yaks were posted in eight intent categories: personal admission, observation, information/advice, opinion, venting/complaining, invitation, favor, and joke. This sets a framework for the intentions of Yik Yak users, but it does not elaborate on the popular topics discussed. Additionally, this approach does not scale to a larger dataset. 
Black et al. (2016) manually categorized 4,000 Yik Yak posts from 42 campuses over 3 days. They found that campus life, announcements, and sex were popular topics. They also found many profanities and rhetorical questions. However, the dataset was quite limited temporally, and their analysis is not scalable.

Koratana et al. (2016) examined public health evidence on Yik Yak. Using Latent Dirichlet Allocation, they modeled topics to find health-related yaks. While their work relates to our research, they do not compare the prevalence of such behaviors across different campuses; they also do not examine any non-health topics. Additionally, we found that automatic clustering methods did not generalize to other yak topics, instead producing noisy and imprecise categorizations.

Using surveys, Ma et al. (2016) found that people are more likely to share information that is intimate and/or negative when they are assured anonymity in online social networks. See also Peddinti et al. (2014) and Correa et al. (2015) for a similar observation. In contrast with tweets, Whisper posts were generally more sensitive and covered a different set of topics. In another analysis of Whisper, Wang et al. (2014) found that anonymous online social communities show high dispersion and low clustering, with little evidence of long-term relationships between users. Xue et al. (2016) studied location privacy in Yik Yak, showing how an attacker can determine the location from where a yak was posted; such an approach could potentially allow an attacker to correlate yaks to specific users.

Our topic analysis approach has two main advantages over the previously published literature. First, other approaches have primarily used labor-intensive manual labeling, which limited the extent of their inferences. Since our approach uses supervised machine learning, it can be automatically scaled to larger datasets. Second, we are not aware of any other research that compared yakking topics across different settings. These two contributions (i.e., scalability and contextbased comparison) are a significant and novel contribution to the body of research on anonymous location-based social networks.

\section{CONCLUSION}

In this article, we investigated a potentially powerful new methodology for making passive regional surveys, namely combining location-based mobile apps with GPS hacking. Although in this article we focused our study on using Yik Yak to survey student sentiment and interest at US college campuses, the general methodology can be employed with other location-based mobile apps. For example, ethic-group centric apps (such as WeChat for Chinese users) can potentially be used to survey diaspora and immigration trends; traffic apps such as Waze can potentially be used to estimate urban development.

We combined Yik Yak with GPS hacking to develop a novel platform for surveying college student interests and sentiment. Using this platform, we collected nearly 1.6 million yaks from a diverse set of 45 college campuses in the United States. We employed an NLP tool to determine the sentiment (positive, negative, or neutral) of all of the yaks. We also used supervised machine learning to classify all the yaks into nine topics and investigate which topics are most popular throughout the US and how topic popularity varies on the different campuses. We have provided examples of yaks that have high positive and high negative sentiment values, examples of the most distinguishing features for gender prediction, and examples of yaks from each of the topics. Some of more interesting findings are as follows:

- At all 45 universities, there are more negative yaks than positive yaks.

- Sentiment ratios vary significantly from campus to campus. However, for many college categories-including Christian schools, liberal arts colleges, big-ten schools, and 
top-ranked schools-Yik Yak sentiment levels are remarkably similar for the colleges within the category.

- Student sentiment is most positive on Yik Yak at Christian universities, followed by liberal arts colleges. Yik Yak sentiment is least positive in two-year colleges.

- Based on the word content of a single anonymous yak, it is possible to predict to gender with a reasonable amount accuracy; some yaks may be predicted with a higher level of confidence. Stylometry, either alone or combined with the bag-of-words approach, does not improve gender prediction precision when using a single yak.

- Aggregated across all the universities, male sentiment ratio and emotion level are about the same as female sentiment ratio and emotion level.

-Dating and sex comprise a large portion of the conversations on Yik Yak. Popular culture and academics are also widely discussed.

- Campuses with higher rates of yakking about dating and sex have lower rates of academically related yaks.

- Schools with lower admission rates and higher SAT scores have lower rates of alcohol and drug-related yaks. Southern schools yak more about alcohol than those from other regions.

Our study has some limitations, however. When predicting gender, we were unable to obtain high levels of precision. This is due to the fact that we are predicting gender using very little information, specifically, using only the contents of a single yak. Many of the yaks provide very few hints about gender. Another limitation is that our topic analysis relies on labeling $2 \%$ of the samples.

Yik Yak's anonymity allows students to express themselves candidly without self-censorship. The methodology in this article can provide significant insight into how student thinking and campus culture vary among US campuses.

In future work, it would be of interest to do a similar study using Twitter and/or Instagram and compare the results with Yik Yak. Twitter and Instagram are not anonymous, so it would be of interest to see if people are being more open and candid in Yik Yak than in other social networks, where users may employ self-censorship. To carry out a similar analysis with Twitter and Instagram, for each university under investigation, one would first have to determine users who are current students at the university, which may lead to some complications.

\section{ACKNOWLEDGMENTS}

The views and conclusions contained in this document are those of the authors and should not be interpreted as necessarily representing the official policies, either expressed or implied, of any of the sponsors.

\section{REFERENCES}

Apoorv Agarwal, Boyi Xie, Ilia Vovsha, Owen Rambow, and Rebecca Passonneau. 2011. Sentiment analysis of twitter data. In Proceedings of the Workshop on Languages in Social Media (LSM'11). Association for Computational Linguistics, 30-38.

Shlomo Argamon, Moshe Koppel, James W. Pennebaker, and Jonathan Schler. 2009. Automatically profiling the author of an anonymous text. Commun. ACM 52, 2 (2009), 119-123.

Ricardo Bilton. February 18, 2016. How BBC is using Yik Yak to talk to millennials (and get them to talk back). Digiday (February 18, 2016)

Erik W. Black, Kelsey Mezzina, and Lindsay A. Thompson. 2016. Anonymous social media-understanding the content and context of Yik Yak. Comput. Hum. Behav. 57 (2016), 17-22.

Denzil Correa, Leandro Araújo Silva, Mainack Mondal, Fabrício Benevenuto, and Krishna P Gummadi. 2015. The many shades of anonymity: Characterizing anonymous social media content. In Proceedings of the International AAAI Conference on Web and Social Media (ICWSM'15). AAAI Press, 71-80.

Diana Graber. May 26, 2014. Yik Yak app makers do the right thing. Huffington Post Screensense Blog (May 26, 2014). 
Aditya Dev Gupta. 2015. Yak Grabber Python Library. Retrieved from https://github.com/adityadevgupta/cs224u-project/ blob/master/YakGrabber.py.

Matthew Heston and Jeremy Birnholtz. 2016. (In)visible cities: An exploration of social identity, anonymity and locationbased filtering on Yik Yak. In iConference. iSchools.

Nathalie Japkowicz. 2000. The class imbalance problem: Significance and strategies. In Proceedings of the 2000 International Conference on Artificial Intelligence (ICAI'00). 111-117.

Abby Kiesa, Alexander P. Orlowski, Peter Levine, Deborah Both, Emily Hoban Kirby, Mark Hugo Lopez, and Karlo Barrios Marcelo. 2007. Millennials Talk Politics: A Study of College Student Political Engagement. Center for Information and Research on Civic Learning and Engagement (CIRCLE) (2007).

Taehoon Kim and Kevin Wurster. 2015. Emoji for Python. Retrieved from https://github.com/carpedm20/emoji/.

Moshe Koppel, Shlomo Argamon, and Anat Rachel Shimoni. 2003. Automatically categorizing written texts by author gender. Liter. Ling. Comput. 17, 4 (2003), 401-412.

Animesh Koratana, Mark Dredze, Margaret S. Chisolm, Matthew W. Johnson, and Michael J. Paul. 2016. Studying anonymous health issues and substance use on college campuses with Yik Yak. In AAAI Workshop on World Wide Web and Population Health Intelligence, Vol. WS-16-15. AAAI Press.

Adam Lella. 2015. Venmo, BuzzFeed, Tinder and Snapchat among the top 20 apps with highest concentration of millennials. comScore (September 24, 2015).

Xiao Ma, Jeff Hancock, and Mor Naaman. 2016. Anonymity, intimacy and self-disclosure in social media. In Proceedings of the 2016 CHI Conference on Human Factors in Computing Systems (CHI'16). ACM, 3857-3869.

Jonathan Mahler. March 8, 2015. Who spewed that abuse? Anonymous Yik Yak app isn't telling. New York Times (March 8, 2015).

Grant McKenzie, Benjamin Adams, and Krzysztof Janowicz. 2015. Of oxen and birds: Is Yik Yak a useful new data source in the geosocial zoo or just another twitter?. In Proceedings of the 8th ACM SIGSPATIAL International Workshop on Location-Based Social Networks (LBSN'15). ACM, 1-4.

Eddy S. W. Ng, Linda Schweitzer, and Sean T. Lyons. 2010. New generation, great expectations: A field study of the millennial generation. 7. Bus. Psychol. 25, 2 (2010), 281-292.

Kathryn M. Northcut. 2015. Dark side or insight? Yik Yak and culture on campus. In Proceedings of the Professional Communication Conference (IPCC'15). 1-5.

National Center of Education Statistics. 2014. Enrollment of the 120 Largest Degree-granting College and University Campuses, by Selected Characteristics and Institution: Fall 2013. Retrieved from https://nces.ed.gov/programs/digest/d14/ tables/dt14_312.10.asp?

G. Paltoglou and M. Thelwall. 2012. Twitter, MySpace, Digg: Unsupervised sentiment analysis in social media. ACM Trans. Intell. Syst. Technol. 3, 66 (2012), 1-19.

Bo Pang, Lillian Lee, and Shivakumar Vaithyanathan. 2002. Thumbs up? sentiment classification using machine learning techniques. In Proceedings of the Conference on Empirical Methods on Natural Language Processing (EMNLP'02). Association for Computational Linguistics, 79-86.

Sai Teja Peddinti, Keith W. Ross, and Justin Cappos. 2014. “On the internet, nobody knows you're a Dog”: a twitter case study of anonymity in social networks. In Proceedings of the 2nd ACM Conference on Online Social Networks (COSN'14). ACM, 83-94.

James W. Pennebaker, Martha E. Francis, and Roger J. Booth. 2001. Linguistic Inquiry and Word Count. Lawerence Erlbaum Associates.

Delip Rao, David Yarowsky, Abhishek Shreevats, and Manaswi Gupta. 2010. Classifying latent user attributes in twitter. In Proceedings of the 2nd International Workshop on Search and Mining User-generated Contents (SMUC'10). ACM, 37-44.

Nathan Rubelke. October 21, 2015. Campuses'symbolically' ban Yik Yak as battle over app rages. The College Fix (October 21, 2015).

Gang Wang, Bolun Wang, Tianyi Wang, Ana Nika, Haitao Zheng, and Ben Y Zhao. 2014. Whispers in the dark: Analysis of an anonymous social network. In Proceedings of the 2014 Conference on Internet Measurement Conference (IMC'14). 137-150.

M. Whissel. 1989. The dictionary of affect in language. In Emotion: Theory Research and Experience. Academic Press, London.

Minhui Xue, Cameron Ballard, Kelvin Liu, Carson Nemelka, Yanqiu Wu, Keith W. Ross, and Haifeng Qian. 2016. You can yak but you can't hide: Localizing anonymous social network users. In Proceedings of the 2016 ACM Internet Measurement Conference (IMC'16). ACM, 25-31.

Received November 2016; revised March 2017; accepted April 2017 